\title{
INTERNATIONAL JOURNAL OF EPILEPSY \\ EVENTS CALENDAR
}

National

* 18th Joint Annual Conference of Indian Epilepsy Association and Indian Epilepsy Society (ECON2017) will be held at Patna (Bihar), India from 17th-19th February, 2017 at Hotel Mourya

International

* Art Competition to celebrate International Epilepsy Day 2017 - Monday 13th February

* 16-20 January 2017, 7th EPODES Advanced II Comprehensive Epileptic Surgery Course Brno, Czech Republic

* 16-18 February 2017, 4th East Mediterranean Epilepsy Congress, Luxor, Egypt

* 2-6 September 2017, 32nd International Epilepsy Congress, Barcelona, Barcelona, Spain

* 12-14 May 2017, 3rd African Epilepsy Congress, Dakar, Senegal

* 21-24 June 2018, 12th Asian and Oceanian Epilepsy Congress, Bali, Indonesia 